\title{
Home Energy Recommendation System (HERS): A Deep Reinforcement Learning Method for Electricity Optimization in Smart Homes
}

This paper was downloaded from TechRxiv (https://www.techrxiv.org).

\section{LICENSE}

CC BY-NC-SA 4.0

SUBMISSION DATE / POSTED DATE

06-09-2021 / 11-09-2021

\section{CITATION}

Shuvo, Salman Sadiq; Yilmaz, Yasin (2021): Home Energy Recommendation System (HERS): A Deep Reinforcement Learning Method for Electricity Optimization in Smart Homes. TechRxiv. Preprint. https://doi.org/10.36227/techrxiv.16574711.v1

$\mathrm{DOI}$ 


\title{
Home Energy Recommendation System (HERS): A Deep Reinforcement Learning Method for Electricity Optimization in Smart Homes
}

\author{
Salman Sadiq Shuvo, Student Member, IEEE, Yasin Yilmaz, Senior Member, IEEE
}

\begin{abstract}
Home appliances that have smart features are becoming increasingly popular. These devices can take command and act intelligently, making them suitable for implementing optimization techniques. Artificial intelligence (AI) based control of these smart devices enables demand-side management (DSM) of electricity consumption. By integrating human feedback and activity in the decision process, this work proposes a deep Reinforcement Learning (RL) method for managing smart devices to optimize electricity cost. Our contributions are twofold. Firstly, we incorporate human feedback in the objective function of our DSM technique that we name Home Energy Recommendation System (HERS). Secondly, we include human activity data in the RL state definition to enhance the energy optimization performance. We perform comprehensive experimental analyses to compare the proposed deep RL approach with existing approaches that lack the aforementioned critical decision-making features. The proposed model is robust to varying resident activities and preferences and applicable to a broad spectrum of homes with different resident profiles.
\end{abstract}

Index Terms-Home energy management, demand side management, electricity optimization, deep reinforcement learning, Markov decision process, artificial intelligence.

\section{NOMENCLATURE}

Devices

AC Air Conditioning

DW Dish Washer

WD Washer and Dryer

EV Electric Vehicle

Parameters at time step $t$

$X_{t}^{i} \quad$ Activity label of the $i$ th resident.

$H_{t}^{i} \quad$ Previous activity labels of the $i$ th resident.

$\omega_{t}^{i} \quad$ Duration of current activity in hours of the $i$ th resident.

$\rho_{t} \quad$ Real-time electricity price in $\$ / \mathrm{kWh}$.

$f_{t}^{d} \in\{0,1\}$, Human feedback for device $d$.

$P_{t}^{d} \quad$ Power consumption in $\mathrm{kW}$ for device $d$.

$\beta_{t}^{d} \in[0,1]$, Battery charge level of device $d$.

Other symbols

$D \quad$ Total number of smart devices.

$d \quad$ Device index.

$\delta_{d} \quad$ Discomfort cost coefficient for device $d$.

$\psi_{d} \quad$ Scheduling time steps for device $d$ (Type-2).

$\mathcal{E}_{d} \quad$ Charging power levels for device $d$ (Type-3).

$\kappa \quad$ Duration of time step in hours.

Both authors are with the Department of Electrical Engineering of the University of South Florida, Tampa, FL 33620, USA ( $\{$ salmansadiq,yasiny $\} @$ usf.edu).

\section{INTRODUCTION}

\section{A. Home Energy Management (HEM) for Smart Homes}

Smart home systems can enhance human comfort and optimize electricity usage in an automated setup. While many devices have included sensor-based control for a long time, such as microwave ovens, air conditioning, etc., with the Internet of Things (IoT) revolution [1], many other smart appliances are entering our homes. Most of the devices will soon have such intelligence that will unlock the true potential of the smart home concept. Specifically, recent smart Home Energy Management (HEM) technologies can leverage stateof-the-art artificial intelligence (AI) techniques. As a result, residents can enjoy all the comfort smart devices offer according to their preferences in an automated way. In addition to personalized comfort, the HEM system can significantly reduce the electricity cost and flatten the demand curve by scheduling some devices to run during off-peak hours.

\section{B. Demand-Side Management (DSM) Techniques for HEM}

Utility companies employ Demand Response (DR) based techniques to encourage customers to shift their load to offpeak hours [2]. It serves two purposes: avoiding electricity purchases from expensive peaking power plants and keeping the system's maximum demand at check to avoid capacity expansion costs. They provide day or hour-ahead dynamic electricity pricing schemes for the customers, known as Time of Use (TOU) [3]. Numerous research has proposed appliance scheduling techniques for HEM systems [4] to capitalize TOU tariffs. Such Demand Side Management (DSM) techniques aim to modify the consumer's energy activities, e.g., shifting customers' electricity usage towards off-peak hours [5]. For instance, [6] proposes a hierarchical HEM system where photovoltaic (PV) energy is integrated into day-ahead load scheduling within a home microgrid. [6] presents a HEM system that further aims to reduce the monthly peak demand to minimize peak demand charges ${ }^{1}$. [7] includes electric vehicle (EV) charging and battery storage in their proposed HEM system. [8] aims to address the uncertainties in electricity usage of smart building HEM as a nonlinear optimization problem. Moreover, [9] proposes a microgrid where the users minimize cost by trading energy between each other before buying from the grid, where PV energy, home battery, and EV battery serve as intermittent sources.

\footnotetext{
${ }^{1}$ Not every utility charges for peak demand.
} 
The majority of the DSM techniques for HEM are based on a rule-based schedule for device usage, undermining consumers' comfort. Rule-based scheduling often suffers from the randomness inherent in human preference, weather, and other interventions, especially in realistic scenarios with multiple residents and multiple appliances. To this end, [10], [11] aim to dissolve the rigid scheduling of devices by including distributed energy generation and distributed energy storage devices in their HEM system. In order to realize the farreaching potential of smart home technology, researchers have opted from conventional mathematical and statistical approaches to recent data-driven machine learning techniques for DSM.

\section{Reinforcement Learning (RL) based DSM techniques}

Electricity consumption patterns are evolving with the fastimproving smart device technologies, which requires adaptability in HEM for scheduling devices. Reinforcement learning (RL) techniques are typically preferred for their datadriven online decision-making capability. Recent advances in neural network based deep RL algorithms lead to widespread applications, including gaming [12], finance [13], transportation [14], communications [15], environmental systems [16], and healthcare systems [17]. [18] provides an extensive review of RL for DSM, showcasing the suitability of RL for DSM techniques. [19] was among the first to investigate RL-based DSM techniques for a smart home. [20] utilizes adaptivity inherent to deep RL algorithms by maintaining thermal comfort and optimal air quality while minimizing electricity usage. The work in [21] provides DSM for households equipped with a smart meter to schedule home devices online. A largescale HEM is proposed in [9] using a multi-agent deep RL framework.

\section{Human Feedback and Activity for HEM}

The authors, in their review of RL for demand response [18], emphasize the importance of incorporating human feedback in RL-based DSM techniques. The work presented in [22] proposes a new smart HEM system in terms of the quality of experience, which depends on the information of consumers' discontent for changing home devices' operations. The work [23] defines human dissatisfaction by the difference between the maximum power rating and the delivered power rating of a device, an oversimplified way of representing human feedback for their RL-based HEM system. [24] calculates dissatisfaction if HEM turns off a device using an equation with different priority factors for different devices. Several other works, e.g., [25], [26], follow a similar approach to estimate discomfort cost rather than using actual feedback from residents. All these techniques lack adaptability to consumer preference, i.e., they may work well for certain types of users, but they are not general enough to ensure user convenience. Accommodating human feedback is challenging since it requires an expensive experimental setup, given that the smart home technology is not widely available in full capacity yet. Park et al. [27] provides theory and implementation for adaptive and occupant-centered lighting optimization in an office setup. They interpret switching on and off the lights by office employees as human feedback. This work has been successful in incorporating human feedback for their RL algorithm, however their scope is limited to lighting. Hence, the necessity for a human feedback-based HEM system still remains open.

The work in [28] proposes a deep sequential learning-based human activity recognition in smart homes. [29] reviews the benefits of labeled activity to analyze and assess the smart home residents' physical and psychological health. [30] analyzes behavior patterns to predict energy consumption profile. Since the smart home concept has the inherent capability of activity labeling, including the activity data as a feature for the DSM technique can greatly facilitate the RL agent's learning capacity.

Although the smart home concept is originally introduced for the residents' benefit, their comfort is often ignored in many existing methods. In this work, we propose a deep RL method that takes the residents' feedback as a reward factor, apart from electricity prices and device status. We consider resident activities as part of system state to better understand human comfort and feedback. Our work incorporates residents' feedback whenever they override the HEM system's commands, a practical and novel way of extending the success of recommender systems (e.g., movie, book, shopping, video) to HEM. Recommender systems learn from customer usage patterns to recommend items/services [31]. A similar approach can be integrated into a HEM system by accommodating human input in a meaningful way.

\section{E. Contributions}

Our contributions lie in addressing two challenges in RL for HEM: integrating human feedback in a meaningful way and utilizing human activity labels appropriately. Specifically, our contributions are:

- a Markov decision process (MDP) formulation for a home energy recommender system (HERS) that incorporates human feedback and activities,

- a deep RL solution to optimize the electricity cost and human comfort,

- comprehensive performance comparisons with a manually controlled, a rule-based, and an RL-based approach [23].

The remainder of the paper is organized as follows. The MDP model is formulated in Section II, and the deep RL algorithm for the optimal policy is given in Section III. The experimental setup is presented in Section IV. Results are discussed in Section V. Finally, the paper is concluded in Section VII with the future research scope.

\section{Model Developement}

We propose an MDP framework shown in Fig. 1, where the smart home device manager is the MDP agent, called HERS. 


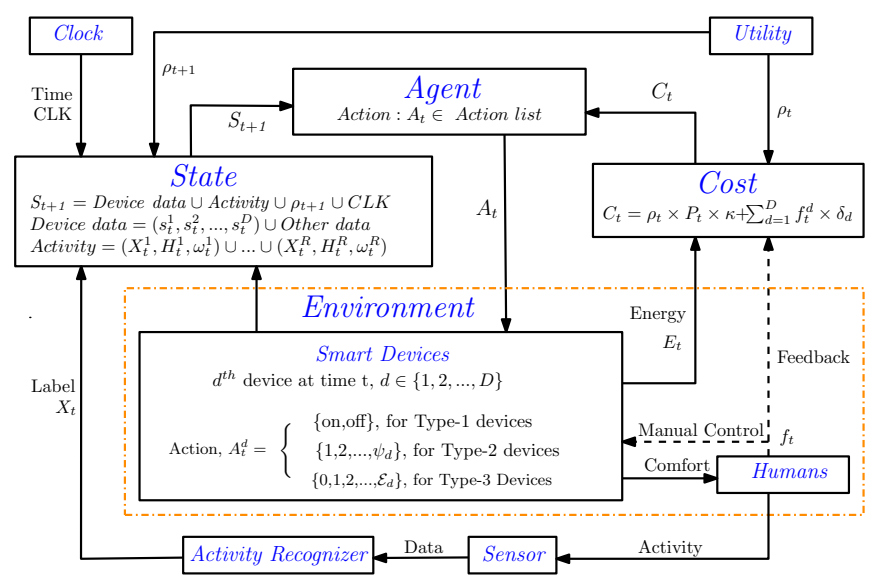

Fig. 1. Proposed MDP model.

\section{A. Environment}

The residents and devices form the MDP environment. The homes can be of different sizes, with multiple residents living in them. We assume access to the utility company's real-time pricing scheme, $\rho_{t}(\$ / \mathrm{kWh}$ at time $t)$. HERS employs different methods to operate each of the $d \in\{1,2, \ldots, D\}$ smart devices that we divide into three categories, as shown in Table I. When switched on by a human or sensor, the device goes into the active status and will be considered for decision-making only during active status.

1) Priority Devices (Type-1): These devices provide essential comfort to the residents, and they are not available for deferring. HERS can keep the active devices off intermittently without compromising the devices' functionality. Regular lights, TV, CCTV camera, alarm system, and air conditioner (AC) are examples of this type of appliance. Choosing the relevant data for the MDP state is a challenge for this task. For instance, if the resident is browsing the internet while the TV is on, turning it off may create discomfort. However, if the resident goes to sleep, keeping the TV on, then turning it off may reduce electricity costs without compromising comfort. $\mathrm{AC}$ is the heaviest load for this device type, hence we focus on it in our experiments.

2) Deferrable Devices (Type-2): These devices can be scheduled later to off-peak hours, reducing electricity cost and maintaining the peak demand lower than the threshold (if any). Dish Washer (DW) and Washer \& Dryer (WD) fall in this category. These devices typically can evade human discomfort if it completes the task before the subsequent activation by the residents. So, the dynamic electricity price $\rho_{t}$ and activation time are critical features for scheduling the deferrable devices.

3) Flexible Devices (Type-3): These devices are flexible in terms of time scheduling and power level. EV, cell phone, and laptop chargers are examples of these types of devices. These devices can consume different power levels $\left\{0,1,2, \ldots, \mathcal{E}_{d}\right\}$, which changes their battery charge level $\beta_{t}$. Residents' activity pattern and $\beta_{t}$ are important features in HERS for these devices.

\section{B. Action, $A_{t}$}

Our MDP model in Fig. 1 starts with the agent selecting actions $A_{t}=\left(A_{t}^{1} \cup A_{t}^{2} \cup \ldots \cup A_{t}^{D_{a c t}}\right)$ about setting the operation
Table I: Device Types

\begin{tabular}{|c|c|c|c|}
\hline Devices & $\begin{array}{l}\text { Priority } \\
\text { (Type-1) }\end{array}$ & $\begin{array}{c}\text { Deferrable } \\
\text { (Type-2) }\end{array}$ & $\begin{array}{l}\text { Flexible } \\
\text { (Type-3) }\end{array}$ \\
\hline $\begin{array}{c}\text { Device } \\
\text { Properties }\end{array}$ & $\begin{array}{l}\text { Not deferrable, Rigid } \\
\text { power consumption }\end{array}$ & $\begin{array}{l}\text { Deferrable, Rigid } \\
\text { power consumption }\end{array}$ & $\begin{array}{l}\text { Deferrable, Flexible } \\
\text { power consumption }\end{array}$ \\
\hline $\begin{array}{l}\text { Operational } \\
\text { Objective }\end{array}$ & $\begin{array}{l}\text { Minimize idle usage } \\
\text { by turning on/off } \\
\text { intermittently }\end{array}$ & $\begin{array}{c}\text { Operate during } \\
\text { low } \rho_{t}\end{array}$ & $\begin{array}{c}\text { Charge highly } \\
\text { at low } \rho_{t}\end{array}$ \\
\hline $\begin{array}{c}\text { Device } \\
\text { Active Status }\end{array}$ & $\begin{array}{c}\text { Turned on by } \\
\text { resident/sensor }\end{array}$ & $\begin{array}{l}\text { Turned on } \\
\text { by resident }\end{array}$ & $\begin{array}{l}\text { Connected to } \\
\text { charger }\end{array}$ \\
\hline $\begin{array}{l}\text { Action } \\
\text { Selection }\end{array}$ & $\begin{array}{c}\text { Every time step } \\
\text { during active period }\end{array}$ & $\begin{array}{c}\text { Once every } \\
\text { activation }\end{array}$ & $\begin{array}{l}\text { Every time step } \\
\text { during active period }\end{array}$ \\
\hline Actions & $A_{t}^{d} \in\{$ on,off $\}$ & $\begin{array}{c}A_{t}^{d} \in\{0,1,2, \\
\left.\ldots, \psi_{d}\right\}\end{array}$ & $\begin{array}{c}A_{t}^{d} \in\{0,1,2 \\
\left.\ldots, \mathcal{E}_{d}\right\}\end{array}$ \\
\hline $\begin{array}{c}\text { Device } \\
\text { Examples }\end{array}$ & $\begin{array}{c}\text { Regular lights, } \\
\text { TV, AC }\end{array}$ & $\begin{array}{l}\text { Sprinkler, } \\
\text { DW, WD }\end{array}$ & $\begin{array}{l}\mathrm{EV}, \text { cell phone, } \\
\text { laptop chargers }\end{array}$ \\
\hline
\end{tabular}

mode for each of the smart devices in active status $D_{\text {act }}(\leq$ $D=m+n+o$ ). So, the total number of possible actions are

$$
\begin{aligned}
m_{\text {Type-1 Devices }}^{2^{m}} & \times \underbrace{\left(\psi_{1}+1\right) \times\left(\psi_{2}+1\right) \times \ldots \times\left(\psi_{n}+1\right)}_{n \text { Type-2 Devices }} \\
& \times \underbrace{\left(\mathcal{E}_{1}+1\right) \times\left(\mathcal{E}_{2}+1\right) \times \ldots \times\left(\mathcal{E}_{o}+1\right)}_{o \text { Type-3 Devices }}, \quad \text { (1) }
\end{aligned}
$$

where, $m$ is the total number of Type- 1 devices. $\psi_{1}, \psi_{2}, \ldots, \psi_{n}$ are scheduling time ranges for the $n$ type- 2 devices, and $\mathcal{E}_{1}, \mathcal{E}_{2}, \ldots, \mathcal{E}_{o}$ are charging power levels for the $o$ type-3 devices. Fig. 2 shows the action flowchart for each type of devices at each time $t$. For the type- 1 devices, there are two actions possible (on/off) for the device. For $A_{t}^{d}=$ off, the agent changes its action if the residents' perform manual override.

HERS keeps a counter $\tau_{d}$ for Type- 2 devices to count the number of steps after the decision-making step for the corresponding device. Initially $\tau_{d}=0$, and HERS schedules the device to run after $A_{t}^{d}$ time steps. While $A_{t}^{d}>\tau_{d}$ and there is no manual override by the resident, HERS keeps the device off and update $\tau_{d}=\tau_{d}+1$. When the counter $\tau_{d}$ reaches the scheduled time, i.e., $A_{t}^{d}=\tau_{d}$, HERS completes the task as scheduled and updates $\tau_{d}=0$. If there is a manual override before the scheduled time, HERS turns the device on to complete the task and updates $\tau_{d}=0$. For Type-3 devices, if there is any manual override, then HERS charge the device at full capacity $\mathcal{E}_{d}$; otherwise implements its decision $A_{t}^{d}$. Every manual override causes the discomfort cost through feedback $f_{t}^{d}=1$ to the RL agent for the corresponding device.

The actual number of possible actions will typically be smaller than Eq. 1 during a time step due to inactive devices. For example, when the residents are not at home, the AC will remain off and will not be considered for the agent's action. Similarly, the idle status of many devices can determined to limit the number of actions. Furthermore, a deferrable device only remains active for one time step when HERS schedules its operation.

\section{State, $S_{t}$}

The MDP agent takes action based on the environment state. Appropriate design of the state is fundamental to the success of the MDP model. As the devices provide comfort to the residents, we hypothesize their activity data to be critical to define the states. An activity recognition system uses various 


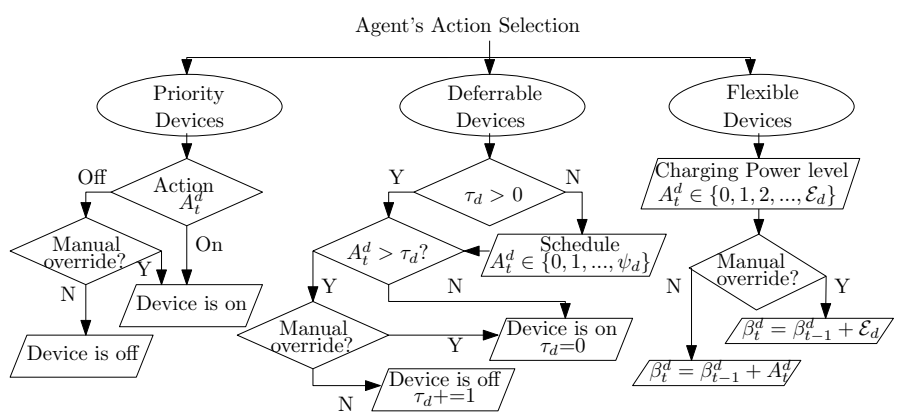

Fig. 2. Action flow chart for active devices at each time $t$.

home sensor data to label the residents' activity $X_{t}$. Apart from the activity, the real-time electricity price $\rho_{t}$ and clock time (CLK) are other essential features that we include in the state definition, as shown in Table II. The state at time $t$ is defined as:

$$
S_{t}=\text { Device data } \cup \text { Activity } \cup \rho_{t} \cup C L K,
$$

where Activity $=\left(X_{t}^{1}, H_{t}^{1}, \omega_{t}^{1}\right) \cup \ldots \cup\left(X_{t}^{R}, H_{t}^{R}, \omega_{t}^{R}\right)$ includes the current activity $X_{t}^{i}$, previous activities $H_{t}^{i}$, and duration of the current activity $\omega_{t}^{i}$ for all $R$ residents. Device data includes information like how long ago the device was activated, the number of dirty dishes or clothes for the Dishwasher and Washer Dryer, the charge level of the type-3 devices, that can be included in the state definition, as shown in Table II. In practice, activity labels can be generated from activity recognition sensors as discussed in [28].

\section{Cost, $C_{t}$}

The MDP agent tries to maximize a reward or minimize a cost by taking optimal actions for a given state. For instance, the RL-based Youtube video recommendation systems are rewarded when the user opens a recommended video [32]. Similarly, HERS receives cost (negative reward) whenever a resident is not happy with the selected action and changes the mode of a device. This human feedback $f_{t}^{d}=1$ is interpreted as discomfort and converted to a cost to the MDP agent through separate cost coefficients $\delta_{d}$ for each device $d$ for each manual override. The devices' operations are meant for human comfort, so HERS' objective is to minimize discomfort.

Total cost for the MDP agent is the sum of energy cost and human discomfort cost. The utility informs the agent of the electricity price for current time step $\rho_{t}$, and future time step $\rho_{t+1}$. The energy usage at time $t$ is obtained from the smart device's power consumption $P_{t}^{d}$ and used to calculate the total cost for each active device for time step $t$ as

Type-1: $C_{t}^{d}=\left(A_{t}^{d} \times P_{t}^{d} \times \kappa \times \rho_{t}\right)+\left(f_{t}^{d} \times \delta_{d}\right)$,

Type-2: $C_{t}^{d}= \begin{cases}\left(P^{d} \times t_{o p}^{d} \times \rho_{t}\right), & \text { if } t \neq t_{o n}^{d}+A_{t_{o n}}^{d}, \\ \left(f_{t}^{d} \times P^{d} \times t_{o p}^{d} \times \rho_{t}\right) & +f_{t}^{d} \times \delta_{d}, \quad \text { otherwise }\end{cases}$

Type-3: $C_{t}^{d}=P_{t}^{d} \times \kappa \times \rho_{t}+f_{t}^{d} \times \delta_{d}$

where $\kappa$ is the unit step time in hours. Cost coefficient $\delta_{d}$ for each device is a critical modeling parameter that converts discomfort into monetary value. $f_{t}^{d}$ represents the discomfort feedback of the residents, where 0 and 1 respectively indicates no override or override. $t_{o n}^{d}$ indicates the index of the decision time step for the Type- 2 device's current operation. $P_{d}$ is the power required for the type- 2 device and $t_{o p}^{d}$ is its operation time. The goal of the MDP agent is to minimize the following discounted cumulative cost for each device in $T$ time steps:

$$
C_{T}^{d}=\sum_{t=0}^{T} \lambda^{t} C_{t}^{d}
$$

where $\lambda \in[0,1]$ is the discount factor for future decisions.

\section{E. Next State, $S_{t+1}$}

At the end of a time step, the device state $S_{t}^{d}$ changes according to the action $A_{t}$; however, human activity data, electricity price data, etc., changes stochastically. These features define the next state $S_{t+1}$, and the dynamic system moves to the next time step for the agent to act. These transitions satisfy the Markovian property of the MDP framework.

\section{SOLUTION APPROACH}

HERS employs one seperate MDP agent for each of the $D$ devices to minimize the discounted total costs $C_{T}^{d}$ in Eq. (3). To achieve the optimal policy arg $\min _{\left\{A_{t}^{d}\right\}} C_{T}^{d}$, we need to solve the following Bellman equation. We drop the device index from here on for brevity. The agent's value function at time step $t$ is

$$
V\left(S_{t}\right)=\min _{A_{t}}\left\{\mathrm{E}\left[C_{t}+\gamma V\left(S_{t+1}\right)\right]\right\} .
$$

The above equation presents a solution dilemma in prioritizing between the immediate $\cos t C_{t}$ and future expected $\operatorname{cost} \gamma V\left(S_{t+1}\right)$. Since the agent's action changes the next state of the devices, the future discounted cost through the value function of the next state $V\left(S_{t+1}\right)$ depends on the action of the agent. Since in high-dimensional problems like the considered one here, it is not feasible to compute the expected future cost explicitly and find the value function for each possible state, deep neural networks are typically used in the modern practice of RL (known as deep RL) to learn the optimal policy of actions either directly (policy-based methods) or through the value function (value-based methods).

The Advantage Actor-Critic (A2C) algorithm, which is a hybrid (both value-based and policy-based) adaptation of policy gradient-based algorithm REINFORCE [33], is a popular choice for continuous state environments. A2C uses the advantage functions for policy update, which reduces the REINFORCE algorithm's variance.

The actor-network, also known as the policy network, outputs probability for each action value through a softmax function. It aims to find the gradient of expected return $J\left(\pi_{\phi}\right)$ of the policy $\pi_{\phi}$ with respect to the weights $\phi$ of the neural network through the following equation:

$$
\nabla_{\phi} J\left(\pi_{\phi}\right)=\mathrm{E}_{\pi_{\phi}}\left[\nabla_{\phi} \log \left(\pi_{\phi}\left(A_{t} \mid S_{t}\right)\right) \mathcal{A}\left(S_{t} ; A_{t}\right)\right],
$$

where the advantage function $\mathcal{A}$ is given by

$$
\mathcal{A}\left(S_{t} ; A_{t}\right)=C_{t}+\gamma V_{\theta}\left(S_{t+1}\right)-V_{\theta}\left(S_{t}\right),
$$




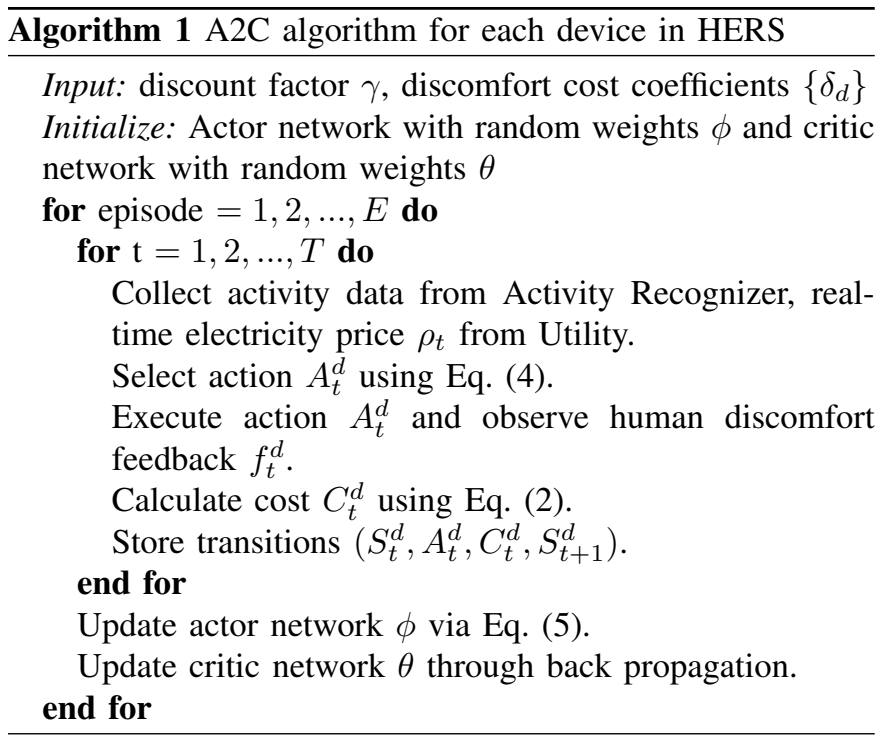

Table II: State Input.

\begin{tabular}{|c|c|c|c|c|c|}
\hline Input & Dimension & AC & DW & WD & EV \\
\hline \hline Activity & 8 & $\checkmark$ & $\checkmark$ & $\checkmark$ & $\checkmark$ \\
\hline Clock time & 1 & $\checkmark$ & $\checkmark$ & $\checkmark$ & $\checkmark$ \\
\hline$\rho_{t}$ & 1 & $\checkmark$ & $\checkmark$ & $\checkmark$ & $\checkmark$ \\
\hline AC status & 1 & $\checkmark$ & $\times$ & $\times$ & $\times$ \\
\hline AC on duration & 1 & $\checkmark$ & $\times$ & $\times$ & $\times$ \\
\hline DW status & 1 & $\times$ & $\checkmark$ & $\times$ & $\times$ \\
\hline $\begin{array}{c}\text { DW activation } \\
\text { duration }\end{array}$ & 1 & $\times$ & $\checkmark$ & $\times$ & $\times$ \\
\hline WD status & 1 & $\times$ & $\times$ & $\checkmark$ & $\times$ \\
\hline $\begin{array}{c}\text { WD activation } \\
\text { duration }\end{array}$ & 1 & $\times$ & $\times$ & $\checkmark$ & $\times$ \\
\hline EV status & 1 & $\times$ & $\times$ & $\times$ & $\checkmark$ \\
\hline Battery charge level & 1 & $\times$ & $\times$ & $\times$ & $\checkmark$ \\
\hline Travel upcoming & 1 & $\times$ & $\times$ & $\times$ & $\checkmark$ \\
\hline EV at-home duration & 1 & $\times$ & $\times$ & $\times$ & $\checkmark$ \\
\hline
\end{tabular}

where $C_{t}$ is the immediate cost. The critic-network learns the value function for each state-action pair. In Eq. (5), $V_{\theta}\left(S_{t}\right)$ is the output of critic network with weight matrix $\theta$. A pseudo code for the proposed $\mathrm{A} 2 \mathrm{C}$ algorithm is given in Algorithm 1.

\section{EXPERIMENTAL SETUP}

The ideal experimental setup would be implementing the HERS algorithm in an existing smart home. However, a fully equipped smart home capable of taking human feedback is yet to be available. We will hypothetically generate human feedback and interactions with the devices based on the residents' activity data. HERS select different features for operating different devices, as shown in Table II. We include clock time in minutes and real-time electricity price $\rho_{t}$ as the common states for all the devices. The New York Independent System Operator (NYISO) provides real-time electricity prices; we use Long Island, NY prices for March 13 and 19, 2021 as the electricity price respectively for weekends and weekdays in our simulation [34]. We find that $\kappa=0.25$ hour (15 minutes) is suitable for the experimental setup.

\section{A. Activity Label}

For residents' indoor activity data, we use the ARAS dataset [35]. The attributes of the dataset for the two homes are shown in Table III. The dataset contains 27 types of activities labeled
Table III: ARAS Activity Dataset [35]

\begin{tabular}{|c|c|c|}
\hline & House A & House B \\
\hline Size & $538 f t^{2}$ & $969 f t^{2}$ \\
\hline Layout & $\begin{array}{l}\text { One bedroom, one living room } \\
\text { one kitchen, one bathroom }\end{array}$ & $\begin{array}{l}\text { Two bedrooms, one living room } \\
\text { one kitchen, one bathroom }\end{array}$ \\
\hline Residents & 2 males at their twenties & Married couple at their thirties \\
\hline Duration & 30 days & 30 days \\
\hline Published in & 2013 & 2013 \\
\hline $\begin{array}{l}\text { Labelled } \\
(5-7) \mathrm{Hay} \\
\text { (10) Sle } \\
\text { (15) Napping } \\
\text { (20) Brushin } \\
\text { (24) Co }\end{array}$ & $\begin{array}{l}\text { ctivities: (1) Going out, (2-4) Coc } \\
\text { g breakfast, lunch, dinner, (8) Wa } \\
\text { ing, (11) Watching TV, (12) Stud } \\
\text { (16) Using Internet, (17) Reading } \\
\text { teeth, (21) Phone conversation, (2 } \\
\text { ersation, (25) Having guest, (26) }\end{array}$ & $\begin{array}{l}\text { ing (breakfast, lunch, dinner), } \\
\text { ing dishes, (9) Having snack, } \\
\text { ng, (13) Bath, (14) Toileting, } \\
\text { ook, (18) Laundry, (19) Shaving, } \\
\text { Listening music, (23) Cleaning, } \\
\text { hanging clothes, (27) Other }\end{array}$ \\
\hline
\end{tabular}

by sensors and validated by the residents themselves. This dataset is comparatively newer and has more activity types than other datasets in the literature. We choose House B for the experiments. HERS takes the current activity label, duration, and the previous two activity labels for each resident (8 inputs in total for the two residents in the house).

\section{B. Devices}

HERS can provide optimal control for all the smart devices in a home. However, we limit our case study to high power loads that renders significant energy cost. Specifically, we choose the following four devices for our experiments.

1) Type-1, Central AC: We estimate a 12000 BTU (3.5 $\mathrm{kWh})$ AC capacity for the $90 \mathrm{~m}^{2}\left(968.75 \mathrm{ft}^{2}\right)$ area of the home, located in a mild temperature zone. In reality, the average AC load is typically half of the capacity [36], so we model the $\mathrm{AC}$ load with the following normal distribution:

$$
P_{A C} \sim \mathcal{N}(\mu=1.8 \mathrm{~kW}, \sigma=0.5 \mathrm{~kW}) .
$$

The AC will be in the idle status $\left(s_{t}^{A C}=0\right)$ if none of the residents are at home or active $\left(s_{t}^{A C}=1\right)$ otherwise. The agent may keep the AC off intermittently under active status; however, the resident will manually turn the $\mathrm{AC}$ on if it causes discomfort. We generate this feedback $f_{t}^{A C}$ if AC goes off within $T_{A C}$ minutes of being turned on. In that case, the residents turn on the AC manually, which penalizes the HERS agent by $\$ \delta_{A C}$. We model $T_{A C}$ with a uniform distribution between 45 to 90 minutes and intermittent off duration as 15 minutes. We include the on duration as a state for AC.

2) Type-2, $D W$ and $W D$ : The activity pattern of house $B$ indicates the lack or no usage of a dishwasher (DW). We generate the dishwashing events to be activated, i.e., $s_{t}^{D W}=1, T_{D W}$ minutes after any resident finishes dinner. We model the delay time $T_{D W}$ with the Poisson distribution with 60 minutes mean value. There will be no dishwashing events for the days when none of the residents have cooked, as there will not be a significant load for the dishwasher. The analysis in [37] estimates 152-minute automatic dishwashing for a comparable load to the considered household. Hence, we model the dishwashing event as a 2.5-hour continuous operation with $1.1 \mathrm{~kW}$ mean and $0.2 \mathrm{~kW}$ variance in power. The Bosch 500 series smart dishwashers are among the most popular models of the year 2020 and serve as the DW model in our experiments [38]. The agent needs to complete dishwashing before the subsequent switching by the residents; otherwise, it receives the discomfort cost $\delta_{D W}$, and the DW is turned on manually to clean the previous dishes. 
Table IV: EV Usage Data Generation.

\begin{tabular}{|c|c|c|c|c|c|}
\hline & \multicolumn{3}{|c|}{ Weekdays } & \multicolumn{2}{c|}{ Weekends } \\
\hline Duration, $t_{a}(\mathrm{hrs})$ & $<8$ & $8-16$ & $>16$ & $<10$ & $>10$ \\
\hline Purpose & Leisure & Office+Leisure & Travel & Leisure & Travel \\
\hline Miles driven, $M$ & $f\left(t_{a}\right)$ & $40+f\left(t_{a}-10\right)$ & n/a & $f\left(t_{a}\right)$ & n/a \\
\hline $\begin{array}{c}\text { Minimum Battery } \\
\text { Before Trip }\end{array}$ & $40 \%$ & $40 \%$ & $70 \%$ & $40 \%$ & $70 \%$ \\
\hline $\begin{array}{c}\text { Battery Status } \\
\text { After Trip }\end{array}$ & $\beta-\frac{M}{220}$ & $\beta-\frac{M}{220}$ & $20 \%$ & $\beta-\frac{M}{220}$ & $20 \%$ \\
\hline
\end{tabular}

House B has a regular heavy load washer \& dryer (WD), so following its laundry schedule would not be practical. The future smart homes will utilize the high-tech WD combos like the LG WM3900HBA, a single compartment light-duty device that takes around 1 hour for washing and 1.5 hours for drying for an average cloth load. We estimate that the residents produce this cloth load every three baths, hence fill and switch the WD in active mode on average 30 minutes (Poisson mean) after their second or third bath (with equal probability) from the previous laundry. Then the RL agent has to turn the WD on for a 1-hour continuous washing cycle, followed by 1.5hour drying cycles with $1.2 \mathrm{~kW}$ mean and $0.2 \mathrm{~kW}$ variance in power to complete the laundry. If the agent does not complete the process before the next switching by the residents, the resident provides negative feedback $\delta_{W D}$ and turns on the WD immediately to clean the previous cloths.

3) Type-3, EV Charging: The residents' activity pattern shows that they mostly go out of the home together. Considering an EV in the house, we assume that the second resident drives it. The EV driver's work pattern seems to consist of long hours with some off days throughout the week. We set his one-way drive to work as 20 miles; 69th percentile driving distance from the data collected by The American Time Use Survey (ATUS) [39], which includes over 13,000 respondents. The activity data provides us with the duration the resident is away from home. Based on the duration, we label such away time as leisure, office time, and travel as in Table IV. We assume the EV is always connected to the charger when the resident is at home.

For weekdays, if the resident stays away for less than 8 hours, it is labeled as a leisure activity, which includes going shopping, visiting friends, short trips, theater, etc. Residents spend more time in leisure activities during the weekend, extending the leisure activity labeling time to 10 hours for the weekend. Driving distance in miles during leisure trip for $t_{a}$ time duration is approximated as;

$$
M=f\left(t_{a}\right)=t_{\text {driving }} \times v_{\text {avg }}=\alpha \times t_{a} \times v_{\text {avg }} .
$$

where, $\alpha=t_{\text {driving }} / t_{a}$ is the ratio of time spent for driving and the total time spent away. We model it with a normal distribution

$$
\alpha \sim \mathcal{N}(\mu=0.33, \sigma=0.1) .
$$

Average speed $v_{\text {avg }}$ is taken as $30 \mathrm{mph}$. The instances in which the resident spends 8-16 hours out of home is labeled as office and leisure activity during weekdays. Round trip to the office is taken as 40 miles, additional time after 10 hours is considered a leisure activity, and driving distance is calculated as $M=40+f\left(t_{a}-10\right)$.

2021 Tesla Model 3 Standard Range is one of the most popular latest EV models with a $450 \mathrm{hp}(336 \mathrm{~kW})$ engine 50

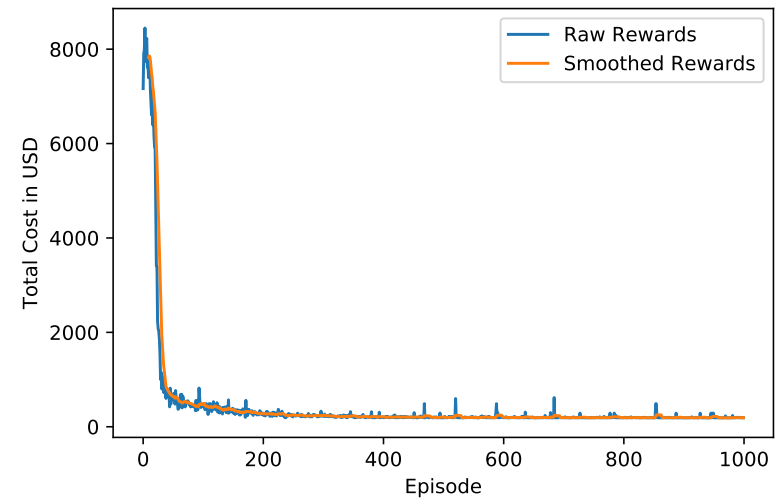

Fig. 3. Convergence of the proposed deep RL algorithm for HERS for scenario-1 total cost.

kWh battery. The level-2 charging of $7.68 \mathrm{~kW}(240 \mathrm{~V} 32$ A) capacity would require 6.5 hours for a full charge of the completely depleted EV battery. Battery status after a trip is the initial battery status when going out of home $\beta$ minus $\frac{M}{220}$ as the Tesla 3 model has a standard driving range of 220 miles. The resident does not use the $\mathrm{EV}$ if $\beta$ is less than $40 \%$ before starting a trip. The resident takes some other transportation mode and assigns a discomfort cost $\delta_{E V 1}$ to the RL agent. If the resident stays more than 16 and 10 hours out of home respectively on weekdays and weekends, we label this activity as travel that may require outside charging. We do not calculate driving distance for traveling; however, we set battery status after the travel to be $5-20 \%$, as home charging is the cheapest and the resident would try outside (paid) charging as little as necessary to reach home. The resident requires a higher initial charge for traveling. We set a higher discomfort cost $\delta_{E V 2}$ if $\beta<70 \%$ before travel. As the initial charge level is higher for travel, we include the next trip type as an input state for the EV.

\section{Discomfort Cost, $\delta_{d}$}

Discomfort cost is a critical parameter in the HERS setup. So, we model it as a user-defined number. Low discomfort cost will emphasize electricity cost, and high discomfort cost will prioritize human feedback. For the experimental purpose, we performed a survey among twenty participants with different backgrounds (e.g., student, homemaker, engineer, etc.) to set the discomfort cost for each of the four devices. Survey results suggest EV charging failure creates the maximum discomfort. Discomfort costs are in decreasing order for WD, $\mathrm{DW}$, and AC. We select discomfort cost coefficients as $\delta_{A C}=$ $20, \delta_{D W}=40, \delta_{W D}=50, \delta_{E V 1}=100, \delta_{E V 2}=300$ in USD.

\section{RESULTS}

We compare our deep RL-based policy with several policies.

(1) Manually controlled policy: In this policy, the residents operate the devices themselves, so a device turns on immediately without any scheduling consideration. We assume the residents turn off the $\mathrm{AC}$ when both of them are out of home 
Table V: Computational statistics for the experiments.

\begin{tabular}{|c|c|c|c|}
\hline Hardware & Software & Task & Computation time \\
\hline Intel(R) Core i7,3.60 & Python 3.7 & LSTM Training & $42 \mathrm{~min}$ \\
\cline { 3 - 4 } GHz, 16 GB RAM & Pytorch 1.8.1 & Online Scheduling & $4 \mathrm{sec}$ \\
\hline
\end{tabular}

and turn it on upon returning. This policy ignores the benefit of smart scheduling, and we will refer to it as the baseline policy to estimate the other policies' success.

(2) Rule-based policy: This policy follows different deterministic rules set by the residents where the device manager is given the day-ahead price of electricity. It schedules the DW and WD during the cheapest time within 12 hours of switching by a resident. EV usage is more random, so the HEM schedules EV only if its battery is more than $50 \%$, otherwise it charges at full capacity when the EV is connected to the charger. AC always stays on if there is any resident at home.

(3) RL-based HEM [23]: In [23], Xu et al. utilize hourahead electricity price as a state to minimize electricity cost. We tailor their approach to fit this comparative analysis with the following modifications: (i) Agent makes decisions every 15 minutes instead of hourly decisions. (ii) There is no PV generation in our setup, so the MDP state consists of electricity price of the next 24 hours, with $4.67 \%$ prediction error following the case-1 (best prediction) in that paper. (iii) We consider the $\mathrm{AC}$ as a priority device that maintains the user set the temperature on its own. Hence, the possible actions for the AC remain turn on or off instead of different power ratings, (iv) We include EV battery depletion, which is overlooked in [23].

Fig. 3 shows that the proposed deep RL algorithm learns the optimal policy within 2000 episodes. Table V shows that training convergence takes 42 minutes and online decision making requires only 4 seconds, exhibiting the real-world applicability. The high cost in the early episodes indicates discomfort among residents; however, it ceases very fast. The trained HERS will take feedback from the consumer to optimize the electricity cost of the house. We examine the above policies for two scenarios.

1) Scenario 1: Unlimited Peak Demand: In this scenario, there is no requirement for keeping the electricity usage within a limit. Fig. 4 shows the daily cumulative cost comparison among policies for different devices and Table VI summarizes the results. The manually controlled policy has the maximum monthly total cost of $\$ 193$; the rule-based policy has the next highest cost with $\$ 185$; [23]'s RL-based approach attains $\$ 185$, and the proposed deep RL-based HERS policy achieves the lowest cost with $\$ 157$. The manually controlled policy starts operation immediately, thus does not take advantage of the lower electricity rate at off-peak hours, unlike the rulebased one. However, the rule-based policy follows a conservative approach for optimization by searching low tariffs in a smaller time window (12 hours for DW and WD) to avoid creating resident discomfort. So, this policy minimizes the cost for all appliances on a smaller scale. The RL-based approach in [23] achieves comparable results with the rule-based policy. The success of this policy is limited due to only including electricity price in its state definition and overlooking many critical features that the HERS policy capitalizes on from (see
Table VI: Monthly Cost Comparison for Different Policies.

\begin{tabular}{|c|c|c|c|c|c|c|c|}
\hline & $\begin{array}{c}\text { Manually } \\
\text { controlled }\end{array}$ & \multicolumn{2}{|c|}{$\begin{array}{c}\text { Rule-based } \\
\text { policy }\end{array}$} & \multicolumn{2}{c|}{$\begin{array}{c}\text { Xu et al. } \\
\text { approach }[23]\end{array}$} & \multicolumn{2}{c|}{$\begin{array}{c}\text { Proposed } \\
\text { HERS }\end{array}$} \\
\hline & Cost (\$) & Cost (\$) & Red. & Cost (\$) & Red. & Cost (\$) & Red. \\
\hline AC & 122.94 & 122.94 & $0 \%$ & 122.94 & $0 \%$ & 99.97 & $18.7 \%$ \\
\hline DW & 5.06 & 3.23 & $36.2 \%$ & 3.19 & $37 \%$ & 3.19 & $37 \%$ \\
\hline WD & 3.27 & 2.35 & $28.1 \%$ & 2.3 & $29.7 \%$ & 2.3 & $29.7 \%$ \\
\hline EV (S1) & 62.01 & 55.99 & $9 \%$ & 56.76 & $8.5 \%$ & 51.6 & $16.8 \%$ \\
\hline Total (S1) & 193.28 & 184.51 & $4.6 \%$ & 185.19 & $4.2 \%$ & 157.06 & $18.7 \%$ \\
\hline EV (S2) & 61.14 & 61.02 & $0.2 \%$ & 60.37 & $1.3 \%$ & 55.88 & $8.6 \%$ \\
\hline Total (S2) & 192.41 & 189.54 & $1.5 \%$ & 188.8 & $1.9 \%$ & 161.34 & $16.1 \%$ \\
\hline
\end{tabular}

Table II). The HERS policy focuses on human feedback in its cost and runs the devices optimally. For instance, HERS keeps the AC off for shorter intervals during midnight without causing any resident discomfort. The proposed deep RL-based policy is expected to decrease the cost further for a system with more devices.

2) Scenario 2: Limited Peak Demand: To avoid overloading a distribution system, the utility company often restricts users to keep energy usage under a threshold. Under this scenario, we limit the peak electricity usage to $10 \mathrm{~kW}$ to obey such restrictions. The EV charging can take up to $7.68 \mathrm{~kW}$ of electricity, even greater than the sum of other loads. So, all the devices other than the EV receive their unrestricted electricity. The EV charging gets the least priority and can consume up to the remaining electricity. Fig. 5 compares the total cost among different policies for both of the scenarios. In Scenario 2, all the policies attain similar results as in Scenario 1 , however with a small increase in cost due to the restrictions. With more devices or lower peak limiting, the results may vary more compared with Scenario 1.

\section{DISCUSSION}

This work focuses on key features derived from residents' activity for operating smart devices. The reward of the RL agent accommodates human feedback, thus provides a setup similar to the popular recommendation systems (e.g., video, book, music, etc.). We understand that any other approach incorporating more customized features for different appliances may achieve further improved results. So, the RL-based recommendation approach for device-specific policy-making has a high potential. This work focuses on demonstrating the benefit of including human activity-based states and human feedback-based rewards for adaptive HEM. Our model provides usage control of devices that do not include PV sources, energy storage, microgrid, and data sharing with other homes or a multi-agent setup. However, our core architecture can accommodate these features in future to open up further research opportunities in this domain.

\section{CONCLUSION}

This work presents a deep Reinforcement Learning (RL) based recommendation system for smart home energy management (HEM). Residents' manual override for a device is interpreted as a negative reward to the RL agent that operates the device. So, the goal of the RL agent is to capitalize lowtariff electricity without creating human discomfort. This is the first work that takes human feedback for device management, to the best of our knowledge. Intuitively, this method works similarly to the popular recommendation applications 

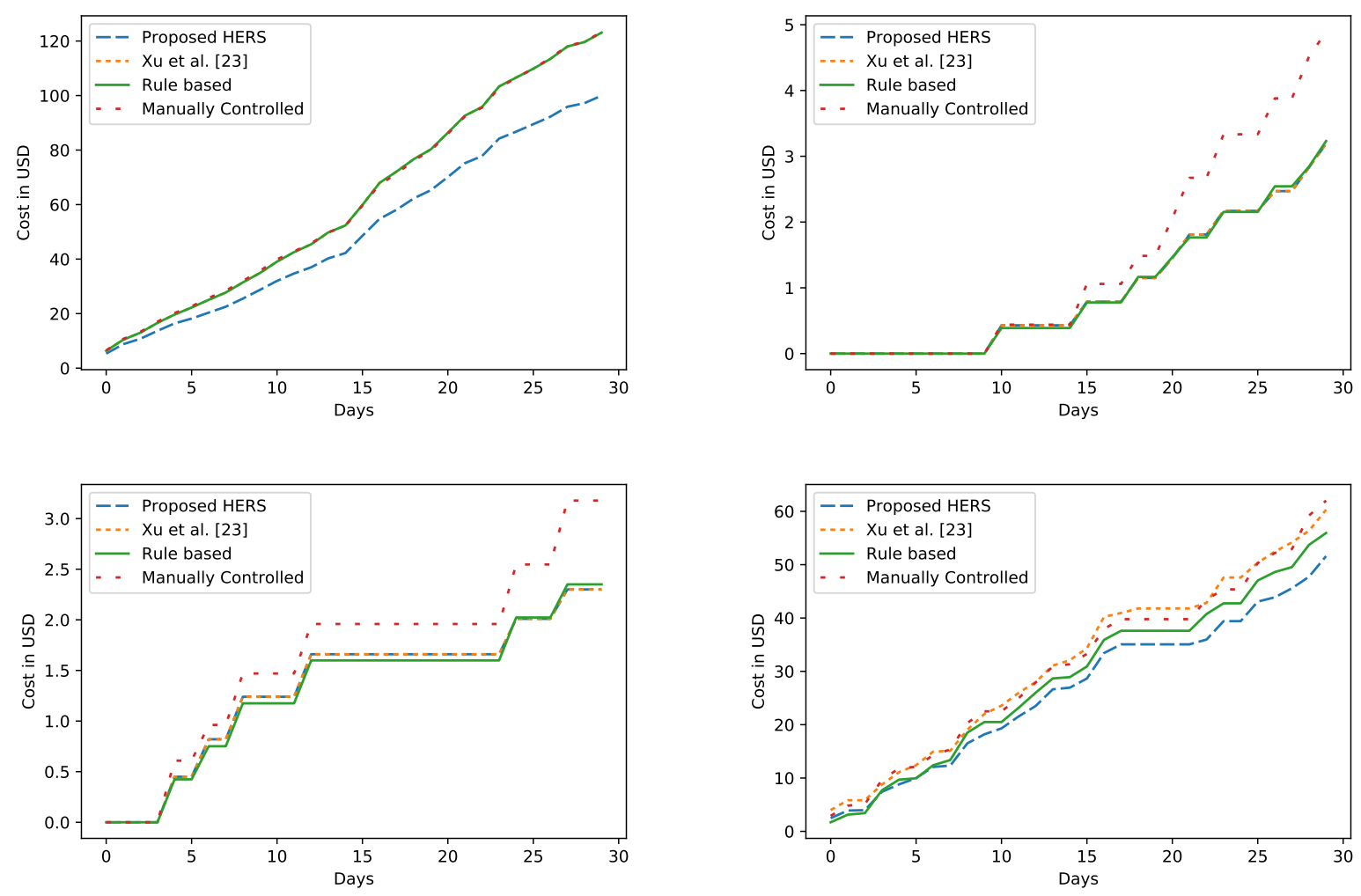

Fig. 4. Daily cumulative cost in Scenario 1 for devices: AC (top left), DW (top right), WD (bottom left), and EV (bottom right) for 1-month duration.
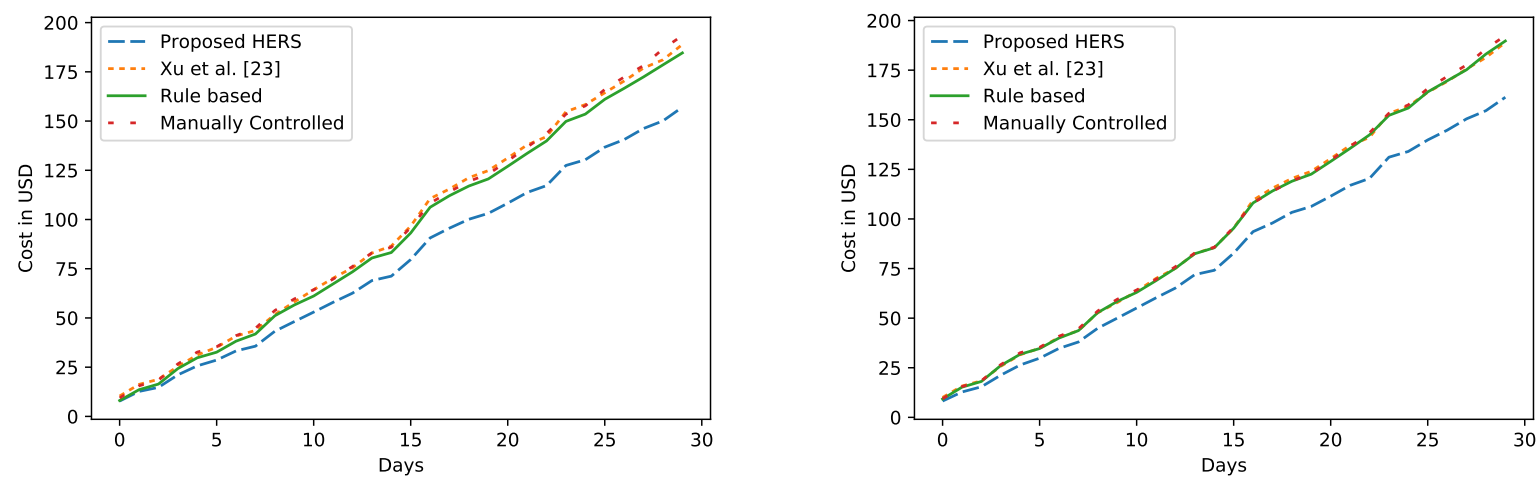

Fig. 5. Daily cumulative cost comparison for all devices among the considered policies for Scenario 1 (left) and Scenario 2 (right).

that suggest video, book, music, etc. based on a user's usage pattern, so we call it Home Energy Recommendation System (HERS). The RL agent considers the human activities for state definition, another novelty the existing literature lacks. The experimental results show that the human activity pattern plays an important role in device operation, in comparison with the $\mathrm{RL}$ approach of $\mathrm{Xu}$ et al. [23] that only considers electricity price for state definition. Our comparative analysis shows that HERS minimizes the electricity cost significantly with respect to the manually controlled policy, rule-based policy, and the RL-based policy presented in [23].

\section{ACKNOWLEDGEMENT}

The authors would like to thank Dr. Hande Alemdar for providing and explaining the ARAS dataset.

\section{REFERENCES}

[1] F. Rocha, L. C. Dantas, L. F. Santos, S. Ferreira, B. Soares, A. Fernandes, E. Cavalcante, and T. Batista, "Energy efficiency in smart buildings: An iot-based air conditioning control system," in IFIP International Internet of Things Conference. Springer, 2019, pp. 21-35.

[2] P. Siano, "Demand response and smart grids-a survey," Renewable and sustainable energy reviews, vol. 30, pp. 461-478, 2014.

[3] H.-T. Roh and J.-W. Lee, "Residential demand response scheduling with multiclass appliances in the smart grid," IEEE Transactions on Smart Grid, vol. 7, no. 1, pp. 94-104, 2015. 
[4] B. Zhou, W. Li, K. W. Chan, Y. Cao, Y. Kuang, X. Liu, and X. Wang, "Smart home energy management systems: Concept, configurations, and scheduling strategies," Renewable and Sustainable Energy Reviews, vol. 61, pp. 30-40, 2016.

[5] G. Strbac, "Demand side management: Benefits and challenges," Energy policy, vol. 36, no. 12, pp. 4419-4426, 2008.

[6] F. Luo, G. Ranzi, S. Wang, and Z. Y. Dong, "Hierarchical energy management system for home microgrids," IEEE Transactions on Smart Grid, vol. 10, no. 5, pp. 5536-5546, 2018.

[7] X. Wu, X. Hu, X. Yin, and S. J. Moura, "Stochastic optimal energy management of smart home with pev energy storage," IEEE Transactions on Smart Grid, vol. 9, no. 3, pp. 2065-2075, 2016.

[8] S. Sharma, Y. Xu, A. Verma, and B. K. Panigrahi, "Time-coordinated multienergy management of smart buildings under uncertainties," IEEE Transactions on Industrial Informatics, vol. 15, no. 8, pp. 4788-4798, 2019.

[9] Y. Yang, J. Hao, Y. Zheng, and C. Yu, "Large-scale home energy management using entropy-based collective multiagent deep reinforcement learning framework." in IJCAI, 2019, pp. 630-636.

[10] W. Li, T. Logenthiran, and W. L. Woo, "Intelligent multi-agent system for smart home energy management," in 2015 IEEE Innovative Smart Grid Technologies-Asia (ISGT ASIA). IEEE, 2015, pp. 1-6.

[11] P. Palensky and D. Dietrich, "Demand side management: Demand response, intelligent energy systems, and smart loads," IEEE transactions on industrial informatics, vol. 7, no. 3, pp. 381-388, 2011.

[12] V. Mnih, K. Kavukcuoglu, D. Silver, A. A. Rusu, J. Veness, M. G. Bellemare, A. Graves, M. Riedmiller, A. K. Fidjeland, G. Ostrovski et al., "Human-level control through deep reinforcement learning," nature, vol. 518, no. 7540, pp. 529-533, 2015.

[13] P. N. Kolm and G. Ritter, "Modern perspectives on reinforcement learning in finance," Modern Perspectives on Reinforcement Learning in Finance (September 6, 2019). The Journal of Machine Learning in Finance, vol. 1, no. 1, 2020.

[14] A. Haydari and Y. Yilmaz, "Deep reinforcement learning for intelligent transportation systems: A survey," IEEE Transactions on Intelligent Transportation Systems, 2020.

[15] A. Nassar and Y. Yilmaz, "Reinforcement learning for adaptive resource allocation in fog ran for iot with heterogeneous latency requirements," IEEE Access, vol. 7, pp. 128 014-128 025, 2019.

[16] S. S. Shuvo, Y. Yilmaz, A. Bush, and M. Hafen, "A markov decision process model for socio-economic systems impacted by climate change," in International Conference on Machine Learning. PMLR, 2020.

[17] H. S. Salman Sadiq Shuvo, Md Rubel Ahmed and Y. Yilmaz, "Deep reinforcement learning based cost-benefit analysis for hospital capacity planning," in International Joint Conference on Neural Networks, 2021.

[18] J. R. Vázquez-Canteli and Z. Nagy, "Reinforcement learning for demand response: A review of algorithms and modeling techniques," Applied energy, vol. 235, pp. 1072-1089, 2019.

[19] H. Berlink and A. H. Costa, "Batch reinforcement learning for smart home energy management," in Twenty-Fourth International Joint Conference on Artificial Intelligence, 2015.

[20] W. Valladares, M. Galindo, J. Gutiérrez, W.-C. Wu, K.-K. Liao, J.-C. Liao, K.-C. Lu, and C.-C. Wang, "Energy optimization associated with thermal comfort and indoor air control via a deep reinforcement learning algorithm," Building and Environment, vol. 155, pp. 105-117, 2019.

[21] J. Lee, W. Wang, and D. Niyato, "Demand-side scheduling based on multi-agent deep actor-critic learning for smart grids," in 2020 IEEE International Conference on Communications, Control, and Computing Technologies for Smart Grids (SmartGridComm). IEEE, 2020, pp. 1-6.

[22] V. Pilloni, A. Floris, A. Meloni, and L. Atzori, "Smart home energy management including renewable sources: A qoe-driven approach," IEEE Transactions on Smart Grid, vol. 9, no. 3, pp. 2006-2018, 2016.

[23] X. Xu, Y. Jia, Y. Xu, Z. Xu, S. Chai, and C. S. Lai, "A multiagent reinforcement learning-based data-driven method for home energy management," IEEE Transactions on Smart Grid, vol. 11, no. 4, pp. 3201-3211, 2020

[24] M. Khan, J. Seo, and D. Kim, "Real-time scheduling of operational time for smart home appliances based on reinforcement learning," IEEE Access, vol. 8, pp. 116520-116534, 2020.

[25] L. Yu, T. Jiang, and Y. Zou, "Online energy management for a sustainable smart home with an hvac load and random occupancy," IEEE Transactions on Smart Grid, vol. 10, no. 2, pp. 1646-1659, 2017.

[26] S. Bahrami, V. W. Wong, and J. Huang, "An online learning algorithm for demand response in smart grid," IEEE Transactions on Smart Grid, vol. 9, no. 5, pp. 4712-4725, 2017.
[27] J. Y. Park, T. Dougherty, H. Fritz, and Z. Nagy, "Lightlearn: An adaptive and occupant centered controller for lighting based on reinforcement learning," Building and Environment, vol. 147, pp. 397-414, 2019.

[28] D. Liciotti, M. Bernardini, L. Romeo, and E. Frontoni, "A sequential deep learning application for recognising human activities in smart homes," Neurocomputing, vol. 396, pp. 501-513, 2020

[29] D. J. Cook, M. Schmitter-Edgecombe, L. Jönsson, and A. V. Morant, "Technology-enabled assessment of functional health," IEEE reviews in biomedical engineering, vol. 12, pp. 319-332, 2018.

[30] C. Chen, D. J. Cook, and A. S. Crandall, "The user side of sustainability: Modeling behavior and energy usage in the home," Pervasive and Mobile Computing, vol. 9, no. 1, pp. 161-175, 2013.

[31] M. Aktukmak, Y. Yilmaz, and I. Uysal, "A probabilistic framework to incorporate mixed-data type features: Matrix factorization with multimodal side information," Neurocomputing, vol. 367, pp. 164-175, 2019.

[32] M. Chen, A. Beutel, P. Covington, S. Jain, F. Belletti, and E. H. Chi, "Top-k off-policy correction for a reinforce recommender system," in Proceedings of the Twelfth ACM International Conference on Web Search and Data Mining, 2019, pp. 456-464.

[33] P.-H. Su, P. Budzianowski, S. Ultes, M. Gasic, and S. Young, "Sampleefficient actor-critic reinforcement learning with supervised data for dialogue management," arXiv preprint arXiv:1707.00130, 2017.

[34] NYISO, "NYISO REAL-TIME DASHBOARD," https://www.nyiso. com/real-time-dashboard.

[35] H. Alemdar, H. Ertan, O. D. Incel, and C. Ersoy, "Aras human activity datasets in multiple homes with multiple residents," in 2013 7th International Conference on Pervasive Computing Technologies for Healthcare and Workshops. IEEE, 2013, pp. 232-235.

[36] A. Burdick, "Strategy guideline: accurate heating and cooling load calculations," National Renewable Energy Lab.(NREL), Golden, CO (United States), Tech. Rep., 2011.

[37] P. Berkholz, R. Stamminger, G. Wnuk, J. Owens, and S. Bernarde, "Manual dishwashing habits: an empirical analysis of uk consumers," International journal of consumer studies, vol. 34, no. 2, pp. 235-242, 2010.

[38] Bosch, "Bosch 500 Series- Stainless steelSHP65T55UC instruction manual," https://media3.bosch-home.com/Documents/9001218494_A. pdf.

[39] M. Muratori, M. J. Moran, E. Serra, and G. Rizzoni, "Highly-resolved modeling of personal transportation energy consumption in the united states," Energy, vol. 58, pp. 168-177, 2013. 